\title{
SYNDICATION
}

\section{Associations between Mycoplasma genitalium, Chlamydia trachomatis and pelvic inflammatory disease}

\author{
I Simms, K Eastick, H Mallinson, K Thomas, R Gokhale, P Hay, A Herring, P A Rogers
}

\begin{abstract}
Objective: To evaluate the association between Mycoplasma genitalium, Chlamydia trachomatis, and pelvic inflammatory disease (PID)

Methods: A case-control methodology was used. Swab eluates were processed using the QIAamp DNA mini kit. Polymerase chain reaction (PCR) for $M$ genitalium was carried out using a real time in-house 16S based assay. An endocervical swab was taken and tested for the presence of $C$ trachomatis (ligase chain reaction, Abbott Laboratories), and a high vaginal swab was taken and tested for the presence of Neisseria gonorrhoeae and bacterial vaginosis.

Results: Of the PID cases 13\% (6/45) had evidence of $M$ genitalium infection compared to none of the controls $(0 / 37) ; 27 \%(12 / 45)$ of the cases had $C$ trachomatis infection compared to none of the controls; and $16 \%(7 / 45)$ of cases only had serological evidence of $C$ trachomatis infection compared to $5 \%(2 / 37)$ of controls. Cases were more likely to present with $M$ genitalium and/or $C$ trachomatis than controls $(\mathrm{p}<0.001)$.

Conclusions: This study indicates that there may be an association between M genitalium and PID, and that this relation is largely independent of $C$ trachomatis. Future studies need to investigate the pathological basis of the relation between $M$ genitalium and PID using samples from women with PID diagnosed using laparoscopy and endometrial biopsy. Little is known about the epidemiology of $M$ genitalium: large scale epidemiological investigations are needed to determine the prevalence, incidence, and factors associated with this emerging infection.
\end{abstract}

See end of article for authors' affiliations

Correspondence to lan Simms;

isimms@phls.org.uk

Accepted for publication 17 October 2002
$\mathrm{P}$ elvic inflammatory disease (PID) is a key issue facing women's reproductive health and has a multifactorial aetiology. Although Chlamydia trachomatis causes a substantial proportion of cases, serological evidence has associated Mycoplasma genitalium with PID. ${ }^{1}$ Previous investigations have been hampered by the lack of simple, accurate diagnostic methods, but polymerase chain reaction (PCR) assays are now available. ${ }^{2}$ A recent Kenyan study suggested an association between $M$ genitalium and acute endometritis. ${ }^{4}$ Here, samples from a case-control study undertaken in England were used to evaluate the relation between $M$ genitalium, $C$ trachomatis, and PID.

\section{METHODS}

A case-control methodology was used which included women aged 16-46 years of age. Patients were derived from a larger case-control study of risk factors associated with PID. ${ }^{5}$ A PCR test for $M$ genitalium that had been developed by the Public Health Laboratory Service became available halfway through the study, and was included in the protocol. Detailed data on sexual behaviour, demographic factors, and reproductive health were collected as part of the larger PID case-control study, but could not be used here because of the small number of $M$ genitalium cases detected and the small overall sample size.

PID cases were diagnosed using the clinical criteria suggested by Hager et al - that is, lower abdominal pain, adnexal tenderness, and tenderness with motion of the cervix and uterus. ${ }^{6}$ This is the standard definition used in departments of genitourinary medicine. Controls were women attending obstetrics and gynaecology for bilateral tubal ligation, and all underwent laparoscopy as part of this procedure. Two endocervical swabs were taken. One was tested for the presence of $C$ trachomatis (ligase chain reaction (LCR), Abbott Laboratories), and the other was tested for the presence of Neisseria gonorrhoeae. A high vaginal swab was taken and tested for the presence of bacterial vaginosis (BV).
Blood samples were also taken to assess serological evidence of chlamydial infection using the microimmunofluorescence (MIF) test. Results of the MIF test were interpreted as follows: $C$ trachomatis titre $=16$, no evidence of infection; titre $\geqslant 32$, evidence of infection; titre of $C$ trachomatis $\geqslant$ titre of $C$ pneumoniae, cross reaction unlikely; titre of $C$ pneumoniae $>$ titre of $C$ trachomatis, indeterminant result.

The $M$ genitalium PCR test used the following protocol. A volume of $200 \mu \mathrm{l}$ of swab eluate was processed using the QIAamp DNA mini kit (Qiagen Ltd) blood and body fluid protocol according to the manufacturer's instructions. For swabs in LCx medium or transport medium $1 \mu \mathrm{g}$ calf thymus DNA was added as a carrier before extraction. Swabs transported to the laboratory dry were incubated at room temperature in 500 $\mu \mathrm{l}$ phosphate buffered saline for 1 hour and vortex mixed. A volume of $200 \mu \mathrm{l}$ of expressed fluid was processed using the QIAamp DNA mini kit swab protocol without carrier DNA. Nucleic acid was eluted from QIAamp columns using $50 \mu \mathrm{l}$ of buffer AE which was incubated on the column for 5 minutes. The same buffer was then used to elute nucleic acid from the column a second time. Amplification was performed by in-house PCR using the LightCycler instrument.

The following primers were used to target the 16S rRNA gene of $M$ genitalium: 16SFG2: 5' CCT TAT CGT TAG TTA CAT TGT TTA A 3' and 16SRG: 5' TGA CAT GCG CTT CCA ATA AA $3^{\prime}{ }^{7}$ Reactions were set up for the LightCycler consisting of (final concentrations in $10 \mu \mathrm{l}$ reactions): $500 \mathrm{nM}$ each primer, $200 \mu \mathrm{M}$ each dNTP, $50 \mathrm{mM}$ TRIS-HCl pH 8.3, $5 \mathrm{mM} \mathrm{MgCl}_{2}$, and 0.65 U Platinum Taq polymerase (prediluted in $1 \mu \mathrm{l} 2.5 \mathrm{mg} / \mathrm{ml}$ BSA to prevent denaturation). This mix was dispensed and made up to $10 \mu \mathrm{l}$ using the QIAamp extracts. The LightCycler instrument (Roche Molecular Systems) was programmed as follows: after initial denaturation of 1 minute at $95^{\circ} \mathrm{C}, 50$

Reproduced in full with permission from Sex Transm Infect 2003;79:154-156. Please use the original citation when citing this article. 
Table 1 Evidence of $M$ genitalium and $C$ trachomatis in cases and controls

\begin{tabular}{lll}
\hline & Cases $(n=45)$ & Controls $(n=37)$ \\
\hline M genitalium & $6^{*}$ & 0 \\
C trachomatis (ligase chain reaction with or without serological evidence) & $12 \dagger \ddagger$ & 0 \\
C trachomatis (serology only) & 7 & 2 \\
Rest & $20 \S$ & 35 \\
\hline *Includes 1 co-infection with C trachomatis. & & \\
tIncludes 1 co-infection with N gonorrhoeae. & \\
$\ddagger 7$ had serological evidence of C trachomatis infection. & \\
$\S 1$ had N gonorrhoeae. & \\
\hline
\end{tabular}

cycles were performed consisting of 0 second at $95^{\circ} \mathrm{C}, 0$ seconds at $55^{\circ} \mathrm{C}$, and 15 seconds at $72^{\circ} \mathrm{C}$ (program type: melting curves). After each cycle a single fluorescence reading was taken. The results were ascertained using a melt cycle at $0.2^{\circ} \mathrm{C}$ per second with continuous fluorescence readings. A positive specimen was judged to have a $\mathrm{T}_{\mathrm{m}}$ within plus or minus $1^{\circ} \mathrm{C}$ of the positive control. This was approximately $88.5^{\circ} \mathrm{C}$. Positive results were confirmed using a hemi-nested block based PCR. $^{3}{ }^{8}$

Statistical analysis was undertaken using the Fisher's exact test (STATA 6). Cases and controls were compared in terms of age using the Mann-Whitney test.

\section{RESULTS}

A total of 82 women were included in the study, 45 with a clinical diagnosis of PID and 37 from patients undergoing tubal ligation. The median age of the cases was 25 (range 16-43), whereas that of the controls was 34 (range 21-45). Cases were significantly younger than the controls $(\mathrm{p}<0.001)$.

Evidence of $M$ genitalium infection was found in 13\% (6/45) of the cases compared to none of the controls, $27 \%$ (12/45) of the cases had $C$ trachomatis infection (LCR with or without serology) compared to none of the controls, and $16 \%$ (7/45) of the cases had only serological evidence of $C$ trachomatis infection compared to $5 \%(2 / 37)$ of controls (table 1$)$. BV was not detected in any of the cases and controls. Two cases had co-infections: one with $C$ trachomatis and $N$ gonorrhoeae, the other between $M$ genitalium and $C$ trachomatis (this patient also had serological evidence of $C$ trachomatis infection). The remaining five $M$ genitalium infected patients had no serological evidence of $C$ trachomatis infection. Of the patients with $M$ genitalium infection, half were over 30 years old. Cases were more likely to present with $M$ genitalium and/or $C$ trachomatis than controls $(\mathrm{p}<0.001)$.

\section{DISCUSSION}

This is the first case-control study of PID to test for $M$ genitalium. The results suggest that there is an association between $M$ genitalium and PID, and that $M$ genitalium is not merely a commensal organism detected at the site of an STI infection. These findings are similar to those from studies of $M$ genitalium in men with non-gonococcal urethritis. ${ }^{9} 10$

There is no standard methodology for sampling the female genital tract for $M$ genitalium. Here, the endocervix was used because it was thought to be the site from which $M$ genitalium migrates to the upper genital tract. The performance of the LightCycler PCR assay was similar to that of the block based assay using the same primers, ${ }^{7}$ but detected fivefold less DNA (equivalent to 10 genome copies) of $M$ genitalium in a dilution series. In tests using 28 common micro-organisms, none produced a product with the $\mathrm{T}_{\mathrm{m}}$ typical of that from $M$ genitalium. However, the testing methodology may lack sensitivity because many of the specimens had previously been tested for $C$ trachomatis using the LCR test which includes an incubation step at $95^{\circ} \mathrm{C}$ in a high magnesium buffer. Anecdotal evidence suggests that mycoplasma DNA may be susceptible to degradation, perhaps due to a relatively low guanine and cytosine content. The inclusion of a serological test would have provided evidence of previous exposure to $M$ genitalium but was not carried out because these specialist techniques were not available at the laboratory.

The Hager definition of PID lacks specificity and consequently some patients included in this study may not have had PID. This problem is inherent to all studies of PID. However, the most crucial element in the design of a case-control study is not to include cases in the control group. Here all controls were laparoscoped to ensure that none of the controls had PID.

The multifactorial aetiology of PID is well established but the public health control of PID has largely centred on the control of genital chlamydial infection. The National Strategy for Sexual Health and HIV recently published by the Department of Health (England) emphasised the need to screen young women for genital chlamydial infection. Although this is a welcome development, this study is a timely reminder that chlamydial intervention alone is unlikely to eradicate PID. The antibiotics used to treat genital chlamydial infection may be less effective in the treatment of $M$ genitalium and, since infection has been shown to be largely independent of $C$ trachomatis, it is likely that the epidemiology of these infections may be different. ${ }^{11}$

Effective PID prevention and control rests on improved knowledge of the pathogenesis and epidemiology of the aetiological agents that cause this clinical syndrome. Further studies need to investigate the pathological basis of the relation between $M$ genitalium and PID using samples from women with PID diagnosed using laparoscopy and endometrial biopsy. This study was based on an opportunistic cohort and it was not specifically designed to examine the relation between $M$ genitalium and PID. A bias within the study was the significant difference in age between the case and control groups. However, since half the patients with $M$ genitalium were over the age of 30 years, this source of bias is unlikely to have significantly influenced the study. Nevertheless, results reported here give an interesting insight into the sequelae associated with $M$ genitalium and provide directions for future studies. Little is known about the epidemiology of $M$ genitalium and specifically designed large scale epidemiological investigations are needed to determine the prevalence, incidence and factors associated with this emerging infection. However, before such studies can be undertaken clear guidelines are needed on the diagnosis of $M$ genitalium and the sites that should be sampled.

\section{ACKNOWLEDGEMENTS}

We would like to thank the British Cooperative Clinical Group. The study was supported by grants from the Department of Health (England) and the Public Health Laboratory Service.

\section{Conflict of interest: none declared.}

\section{CONTRIBUTORS}

IS initiated and designed the project and undertook the analysis with PR; KE and AH developed the $M$ genitalium PCR and did the testing; HM undertook the LCR and serological testing; $\mathrm{KH}, \mathrm{RG}$, and $\mathrm{PH}$ recruited cases and controls for the study. 


\section{Authors' affiliations}

I Simms, Communicable Disease Surveillance Centre, UK

K Eastick, A Herring, Bristol PHL, UK

H Mallinson, Liverpool PHL, UK

K Thomas, Liverpool Women's Hospital, Liverpool, UK

R Gokhale, Arrowe Park Hospital, Wirral, UK

P Hay, St George's Hospital, London, UK

P A Rogers, PHLS Statistics Unit, UK

\section{REFERENCES}

1 Moller BR, Taylor-Robinson D, Furr PM. Serological evidence implicating Mycoplasma genitalium in pelvic inflammatory disease. Lancet $1994 ; 1: 1102-3$

2 Jensen JS, Uldum SA, Søndergård-Andersen J, et al. Polymerase chain reaction for detection of Mycoplasma genitalium in clinical samples. $J$ Clin Microbiol 1991;29:46-50.
3 Palmer HM, Gilroy CB, Furr PM et al. Development and evaluation of the polymerase chain reaction to detect Mycoplasma genitalium. FEMS the polymerase chain reaction to de

4 Cohen CR, Manhart LE, Bukusi EA, et al. Association between Mycoplasma genitalium and acute endometritis. Lance 2002;359:765-6.

5 Simms I, Mallinson H, Peeling RW, et al. Risk factors associated with pelvic inflammatory disease: a UK study. Int J STD AIDS 2002;13(S1): 18 .

6 Hager W, Eschenbach D, Spence $M$, et al. Criteria for diagnosis and grading of salpingitis. Obstet Gynecol 1983;61:113-14.

7 Eastick K, Leeming JP, Caul EO, et al. A novel polymerase chain reaction assay to detect Mycoplasma genitalium. J Clin Pathol (in press). Deguchi T, Gilroy CB, Taylor-Robinson D. Comparison of two 8 Deguchi T, Gilroy CB, Taylor-Robinson D. Comparison of two
PCR-based assays for detecting Mycoplasma genitalium in clinical PCR-based assays for detecting Mycoplasma genitalium in clinico
specimens. Eur J Clin Microbiol Infect Dis 1995;14:629-31.

9 Jensen JS, Orsum R, Dohn B, et al. Mycoplasma genitalium: a cause of male urethritis? Genitourin Med 1993;69:265-9.

10 Totten PA, Schwartz MA, Sjostrom KE, et al. Association of Mycoplasma genitalium with nongonococcal urethritis in heterosexual men. Infect Dis 2001;183:269-76

11 Taylor-Robinson D Horner PJ. The role of Mycolasma genitalium in non-gonococcal urethritis. Sex Trans Infect 2001;77:229-31.

\section{$\mathrm{ECHO}$}

\section{Quality and use of synovial fluid tests open to question}

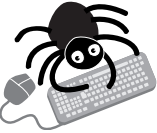

Please visit the Journal of Clinical Pathology website [www. jclinpath.com for link to this full article.
$T$ he use of synovial fluid tests gives cause for concern, despite their obvious diagnostic value in certain joint diseases, suggests a comprehensive literature review of their applications from the University of Bristol.

The review, based on 300 papers out of an initial trawl of 6556, published between 1980 and 2001, confirms that synovial fluid analysis can be of major diagnostic value. It is especially useful in acute arthritis, when a crystal arthropathy or septic arthritis is suspected, and in intercritical gout.

But standards fall well short of optimal for the traditional assays of microbiology, white blood cell counts, and microscopy for pathogenic crystals. And there are "worrying variations" in reported sensitivity, specificity, and reliability, and scant evidence of quality control, it finds.

Furthermore, the use of the newer cytological and biochemical marker assays is based primarily on anecdotal evidence, with no research into their sensitivity, specificity, and reliability, it says. Added to which, the well known difficulties of interpreting the results of biochemical assays restrict their use in clinical medicine.

The authors point out that the poor standards highlighted by the review may be partly due to the fact that synovial fluid analysis is routinely under researched, excluded from routine diagnostic pathology services, and is a victim of low throughput of samples in most units.

More research is urgently needed, say the authors, who conclude that the immediate priority should be to rationalise the use, and improve the quality control, of synovial fluid assays, given their importance in acute arthritis.

A Ann Rheum Dis 2002; 61:493-498 